\title{
Implementation of Data Transmitted Network Base on OPNET
}

\author{
Juan Wang, Lan Qiu \\ Department of information engineering, \\ JiangXi University of Technology, \\ NanChang, 330098, China
}

\begin{abstract}
The paper focuses on research the solution of establishing the model of packet transmitted network and implement of it, withing the powerful simulation of network modeling of OPNET. To see the effect of the packet generation rate on the performance of the network, using the Simulation Editor to create five simulation sets for different packet interarrival time.
\end{abstract}

Keywords-system simulation; optimal network engineering tools; packet; line; end-to-end delay.

\section{OVERVIEW}

Network simulation technology is a kind of technology that including a statistical modal through constructing network equipment and network links and simulate the transmission of network traffic to obtain the required date[1]. The statistical multiplexing randomness is accurately reproduced as the simulation is based on a statistical model rather than on mathematics.

The most famous simulation software is a production of MIL3 in the U.S [2]. So far, OPNEL has been the most advanced development and application platform in the world and it has been selected to be the top one by the third party authority such as Network World. It uses a discrete event-driven simulation mechanism, where "event" refers to changes in network status, that is, only when the network state changes, analog machines are able to work. Any stimulation calculation will not be performed if the state of network doesn't change, i.e., skipped. Therefore, compared with the time-driven, discrete event-driven simulation computer works efficiently. OPNET uses simulation on packet level and its model is divided into three levels, network, node and process in which users can cut programming anywhere to establish the required model.

\section{THE IMPORTANCE OF OPNET MODELER}

OPNET Modeler is a large package, which not only supports network modeling and simulation in general sense but also provides special support for a variety of special networks. Its importance is listed as follows:

Using a hierarchical structure modelling [3]: first, from a network object perspective, three modeling mechanism are provided including an underlying processing model and the agreement will be describe by FSM; second, the node model followed by the corresponding agreed model can reflect the service characteristics; the upper layer is network model corresponding with the actual network and reflecting the network-related features. Three-layer models correspond with the actual agreement, devices, networks exactly and fully reflect the relevant characteristics of the network. As shown in Figure 1.

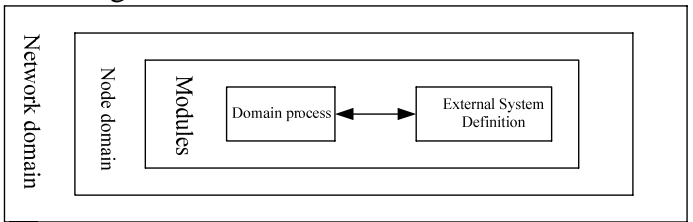

Figure 1. Hierarchical modeling

FSM mechanism: compared with the time driven mechanism, calculating efficiency is greatly improved by using discrete event-driven simulation mechanism.

Use object-oriented modeling mechanism: each type of node is developed by using object-oriented mechanism and the model is generated in application for different objects with specific parameters, as a result the efficiency and utilization are improved

Use hybrid modeling mechanism: combine packed based method with statistical-based method and you will get very detailed simulation results.

Full support for core programming. Completely open system.

\section{THE PROCESSES OF OPNET MODELER}

In this paper, the process of network stimulation by using OPNET includes the following steps[4], As shown in Figure 2.

Determine the objects and issues to be dealt with; Establish the appropriate the topology according the objects and issues, the topology types, background and object family, network rang and size included; Collect and determine statistics, such as a network Serve Load, Load bits and Delay; Save selected items; Operate; Analyze the simulation result. 


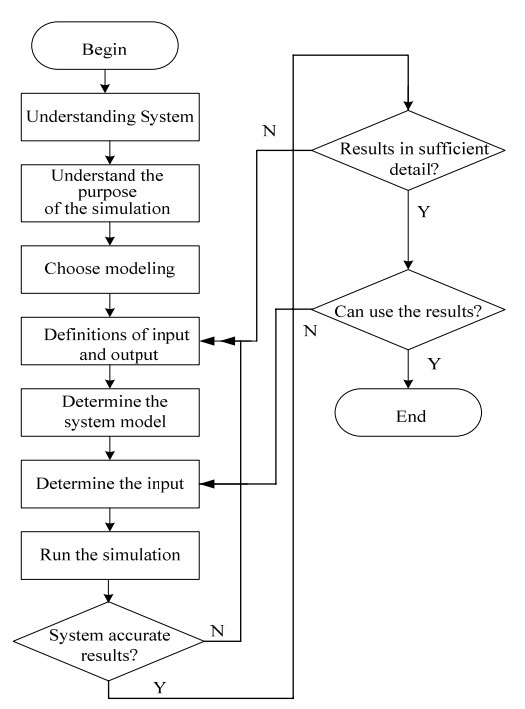

Figure 2. The process of OPNET Modeler

\section{MODEL CONSTRUCTING}

In this paper, Network topology consists of a central node connected to eight peripheral node posed [5].Hub is the central node,node_0 to node_7 is a peripheral node. OPNET software comes with a global map, so the address can be set according to user needs. This paper set up this network in Europe. Central node located in Toronto, and peripheral nodes are located in seven other cities: Pittsburgh, Cleveland, Indianapolis, Chicago, Boston, New York, Philadelphia, Washington. First, the packet generated by the peripheral node is sent to the central node Toronto, and central node analyzes the destination address of the packet, then send it to node referring to the destination address. As shown in Figure 3.

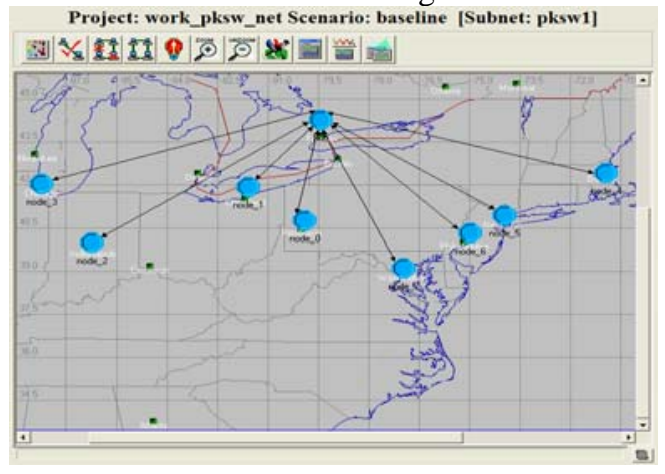

Figure 3. Framework project to be established

Three modeling mechanism of central node. First, central node need eight transceivers, and can process the data of receive and transmit, and then need a process module to accomplish process the data. Central node model is shown in Figure 4.

Central node analyzes destination address of the received data packet, and then sends it to the corresponding data transmitter, last the transmitter transfer the destination address to the corresponding peripheral node [6].All of these tasks from the central node is completed by process module. Process module use a process model of finite state machines, it is included in the node editor, so you can edit the state transition diagram and the corresponding code in the process model editor, and finally let node models of editor process include the process module.

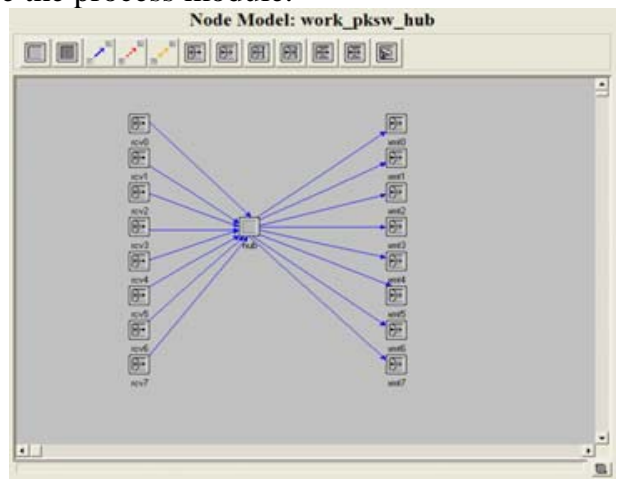

Figure 4.Central node model

\section{SIMULATION RESULTS AND ANALYSIS}

In this simulation, in order to observe the impact of different packet generation rate for the network performance, you need to configure multiple values for the corresponding properties in the simulation really editor, different packet arrival time interval to create five different simulation [7] in the pksw subnet, the Simulation of link utilization select node_0 <-> hub link which from Pittsburgh to Toronto. the size and receiver / transmitter of Rate packet is constant, so it should be fixed for delay from end to end [8].However, if generation rate of the packet is fast enough, it will cause some packages overstock in the transmitter queue, as a result ,packet delay on end to end will increased. If the packet generation rate is uncertain, it may cause traffic bursts, so the delay also affected. To simulate these behaviors, you need to configure simulation property on the source interarrival time, and give it five specified the value, you can run five simulations evey time, each simulation corresponds to a value of the property. Therefore it can get link utilization and end to end delay on different packet transmission rates. Set the packet arrival interval pksw. *. Source interarrival time is 5 seconds, 10, 15 and 25 seconds, the total simulation time is 0.5 hours.

Under different configurations condition, observe link utilization of node_0 to hub. As can be seen from the figure 5 , When sending a data packet interval is 5 seconds, the link utilization is the highest, with the increase of the interval to send packets, the link utilization is successively reduced, when the interval is 25 seconds, which link utilization is the lowest. In this paper, the simulation of the link utilization is consistent with the theory that the rate slowed down when the link utilization is significantly reduced, which means reducing the amount of data packets and the corresponding lowering of the link utilization. 
When packet size and the rate of receiver / transmitter is constant, delay on end to end should be fixed. Shown in Figure 6, delayed image according to different transmission interval time of data packet, under the five configurations condition, the end to end delay in the simulation carried out immediately when the data is relatively large, when the system reaches a steady, the delay is also gradually stabilized, five-end delay simulation curve of the general trend is consistent.

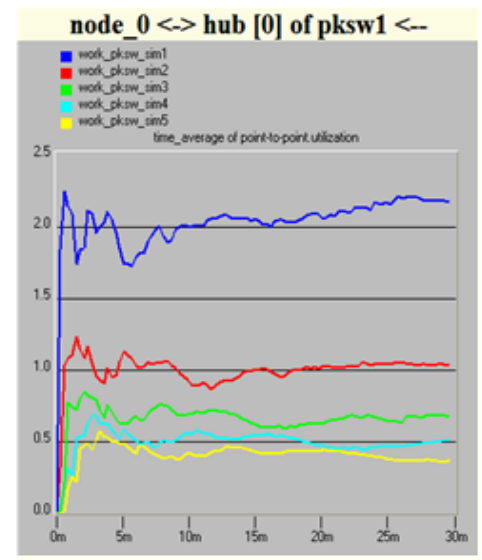

Figure 5. The displays of the link utilization

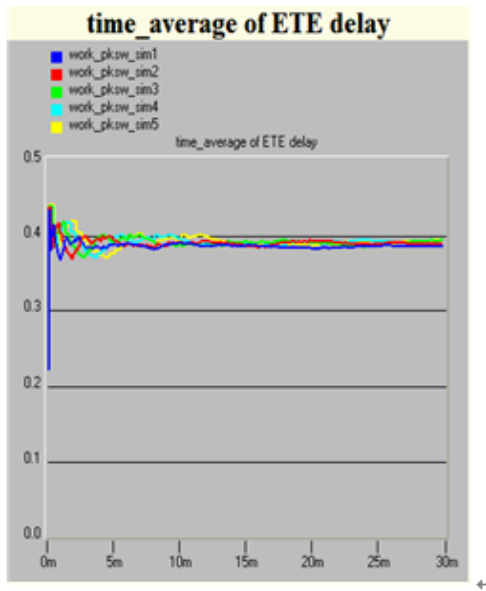

Figure 6. The displays end to end delay

Packet generator creates constant size packets, combine with the fixed data transmission rate of transmitter and receiver from end to end, each packet will have a fixed end to end delay .However, if sending rate of 12. the transmitter is large enough, some packets will be blocked in the transmitter queue, which will cause effect to end to end delay. When transmission rate of the packet increase , the delay aslo increased to certain extent, and the most of $t$ data packet of end delay is relatively constant, but there are still a part of the packet has experienced higher-end latency.

\section{CONCLUSION}

OPNET is an excellent communication protocol modeling and simulation tool[9], which is currently the world's most advanced network emulation and application platform, it has advanced modeling mechanism, the advantages of a comprehensive model library, complete external interface, through more subnet nested layer to achieve complex network topology management, modeling provides three mechanisms, thus OPNET has been quite widely used.

\section{REFERENCES}

[1] Hosein, Michael A.;Seegobin, Rhea L. Transaction Processing and Distribution with COPAR and Opnet[J]. GSTF Journal on Computing. 3(4),pp.122-126,2014.

[2] Seegobin, Rhea L.;Hosein, Michael A. Using Opnet Modeller for the Delivery of the COPAR Service and Improved Availability on the Internet[J]. GSTF Journal on Computing. 3(4),pp.82-89,2014

[3] Ling Zhao, Jianhua Liu.OPNET network simulation technology and its application [J]. Microcomputer Information, vol. 13, pp. 186-188, 2010.

[4] Lin Zhao, Ling Song, Caixia Xiao. Based on Campus Network Performance Analysis and Research Opnet simulation [J]. Popular Science, vol. 11, pp. 28-29, 2010.

[5] Mohammed Hawa. An OPNET simulation model for peer-to-peer networks[J].SIMULATION. Vol.89,pp.1009-1019,2013.

[6] Fangtian Hou;Cheng Yang;Jianbo Liu;Yichun Zhang;Jiayin Tian;Yakun Zhang. An analysis of HB-MAP based on OPNET simulation [J]. Wuhan University Journal of Natural Sciences, Vol.18,pp.49-54,2013

[7] YaTao Yang, ZhiWei Chen, SaiNan Zhou. UMTS network simulaiton and performance ananlysis based on OPNET[J]. Journal of Beijing Electronic Science and Technology Institute, vol.4,pp.26-29,2012.

[8] Yin Chen, Yang Song, MinRui Fei. Design and development of cosimulation platform for NCS based on simulink and OPNET[J] - Journal of System Simulation, vol.7,pp. 1518-1523,2013.

[9] MITTAL S.OPNET:An integrated design paradigm for simulation[J]. Software Engineering:An International Journal(SEIJ), vol.2,pp.57-67,20 